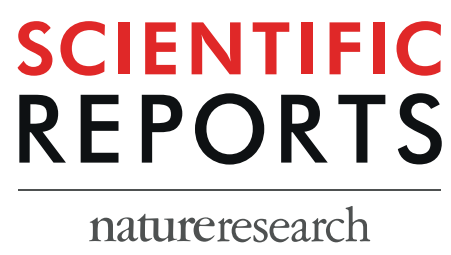

\title{
OPEN Preoperative Neutrophil to Lymphocyte Ratio and Platelet to Lymphocyte Ratio are Associated with the Prognosis of Group 3 and Group 4 Medulloblastoma
}

Received: 19 November 2018

Accepted: 29 August 2019

Published online: 13 September 2019
Ke Li ${ }^{1}$, Wen-chao Duan ${ }^{1}$, Hai-biao Zhao ${ }^{1}$, Li Wang ${ }^{2}$, Wei-wei Wang ${ }^{2}$, Yun-bo Zhan ${ }^{1}$, Tao Sun ${ }^{1}$, Feng-jiang Zhang ${ }^{1}$, Bin Yu ${ }^{1}$, Ya-hui Bai ${ }^{1}$, Yan-min Wang ${ }^{1}$, Yu-chen $\mathrm{Ji}^{1}$, Jin-qiao Zhou ${ }^{1}$, Xian-zhi Liu ${ }^{1}$, Zhi-feng Zhang ${ }^{1}$ \& Zhen-yu Zhang ${ }^{1}$

Inflammation and immunoreaction markers were correlated with the survival of patients in many tumors. However, there were no reports investigating the relationships between preoperative hematological markers and the prognosis of medulloblastoma (MB) patients based on the molecular subgroups (WNT, SHH, Group 3, and Group 4). A total 144 MB patients were enrolled in the study. The differences of preoperative hematological markers among molecular subgroups of MB were compared by One-way ANOVA method. Kaplan-Meier method was used to calculate the curves of progression free survival (PFS) and overall survival (OS). The comparison of survival rates in different groups were conducted by the Log-rank test. Multivariate analysis was used to evaluate independent prognostic factors. Increased preoperative NLR (neutrophil-to-lymphocyte ratio, PFS, $P=0.004,0 S, P<0.001$ ) and PLR (platelet-to-lymphocyte ratio, PFS, $P=0.028,0 S, P=0.003$ ) predicted poor prognosis in patients with MB, while preoperative MLR (monocyte-to-lymphocyte ratio), MPV (mean platelet volume), PDW (platelet distribution width), and AGR (albumin-to-globulin ratio) were revealed no predictive value on the prognosis of patients with MB. Furthermore, high preoperative NLR and PLR predicted unfavorable prognosis in childhood MB patients. However, preoperative NLR and PLR were not associated with the prognosis in adult MB patients. Multivariate analysis demonstrated preoperative NLR (PFS, $P=0.029, \mathrm{OS}, P=0.005$ ) and PLR (PFS, $P=0.023, \mathrm{OS}, P=0.005$ ) were the independent prognostic factors in MB patients. Emphatically, the levels of preoperative NLR and PLR in Group 3 MB were significantly higher than those in WNT MB. High preoperative NLR was associated with unfavorable OS in Group $3(P=0.032)$ and Group $4(P=0.027)$ tumors. Similarly, increased preoperative PLR predicted poor PFS $(P=0.012)$ and OS $(P=0.009)$ in Group 4 tumors. Preoperative NLR and PLR were the potential prognostic markers for MB patients. Preoperative NLR and PLR were significantly associated with the survival of Group 3 and Group 4 tumors.

Medulloblastoma (MB) is one of the most common brain malignant tumors in children, which accounts approximately $20 \%$ of all the pediatric brain tumors ${ }^{1}$. Although the prognosis of patients with MB has been improved by the advancement radiation therapy and chemotherapy, the survival rates of patients with $\mathrm{MB}$ remain considerably different. Exploring new markers to accurately predict the prognosis of patients with MB could contribute to the evaluation and management of the disease.

Recently, more and more studies demonstrated that the preoperative hematological markers played important roles in predicting prognosis of several types of tumors, such as esophageal cancer, colorectal cancer, renal cell

${ }^{1}$ Department of Neurosurgery, The First Affiliated Hospital of Zhengzhou University, Zhengzhou, Henan, 450052, China. ${ }^{2}$ Department of Pathology, The First Affiliated Hospital of Zhengzhou University, Zhengzhou, Henan, 450052, China. Ke Li, Wen-chao Duan and Hai-biao Zhao contributed equally. Correspondence and requests for materials should be addressed to Z.-f.Z. (email:zzfh72@sina.com) or Z.-y.Z. (email:neurozzy@foxmail.com) 
carcinoma, and glioblastoma, etc. ${ }^{2-5}$. Specifically, increased preoperative neutrophil-to-lymphocyte ratio (NLR), platelet-to-lymphocyte ratio (PLR), and monocyte-to-lymphocyte ratio (MLR) were reported poor prognosis in patients with solid cancers ${ }^{6-8}$. Reduced preoperative mean platelet volume (MPV) was discovered in patients with renal cell carcinoma compared to the patients with benign renal tumor ${ }^{9}$. Moreover, preoperative platelet distribution width (PDW) was demonstrated as an independent risk factor for the prognosis with gastric cancer ${ }^{10}$. However, to our knowledge, no studies have investigated the prognostic significance of preoperative hematological markers in $\mathrm{MB}$ patients.

The determination of molecular subgroups was one of the most important advancements in the realm of MB investigation. Mounting evidences demonstrated that MB is a heterogenous disease and composed of different molecular subgroups: sonic hedgehog (SHH), wingless (WNT), Group 3, and Group 4. These subgroups are significantly different in transcriptional profiles, genetic abnormalities, clinical characteristics, and prognosis ${ }^{1,11}$. Recently, the four MB molecular subgroups have been included in the newest 2016 World Health Organization classification of tumors of the central nervous system ${ }^{12}$. Nevertheless, whether the prognostic value of hematological markers differ in molecular subgroups of MB remains unexplored.

The purpose of the present study was to investigate the prognostic significance of the preoperative hematological markers (NLR, PLR, MLR, MPV, PDW, and albumin-to-globulin ratio (AGR)) combined with molecular subgroups (WNT, SHH, Group 3, and Group 4) on the survival of patients with MB.

\section{Results}

Clinicopathologic characteristics. $144 \mathrm{MB}$ patients had complete preoperative hematological markers without preoperative infection diseases and systemic comorbidities were enrolled in the study (Supplementary Fig. 1), including $25 \mathrm{WNT}, 30 \mathrm{SHH}, 51$ Group 3, and 38 Group $4 \mathrm{MB}$. The mean preoperative NLR for WNT, $\mathrm{SHH}$, Group 3 and Group $4 \mathrm{MB}$ were $1.56 \pm 0.84,2.19 \pm 1.81,4.02 \pm 3.77$, and $3.31 \pm 5.50$. The mean preoperative PLR for WNT, SHH, Group 3 and Group $4 \mathrm{MB}$ were $110.46 \pm 51.28,130.28 \pm 74.98,163.66 \pm 74.47$, $167.85 \pm 138.77$. The mean preoperative MLR, MPV, PDW, and AGR for $144 \mathrm{MB}$ patients were $0.27 \pm 0.45$, $8.55 \pm 1.42,15.68 \pm 1.42,1.93 \pm 0.44$, respectively (Supplementary Table 1). Clinicopathologic data of the 116 MB patients were successfully followed up exhibited in Tables 1 and 2.93 (80.2\%) patients were children and $23(19.8 \%)$ cases were adults. The mean age of the cohort was 12 years (ranged from 0.9 to 50 years). 75 (64.7\%) patients were male and $41(35.3 \%)$ were female. $79(68.1 \%)$ patients' preoperative karnofsky performance status scale (KPS) $\geq 80$ and 37 (31.9\%) patients' preoperative KPS $<80$. Furthermore, $88(75.9 \%)$ tumors located in midline, and $28(24.1 \%)$ tumors located in lateral. All the tumors underwent resection: $62(53.4 \%)$ tumors had gross total resection, 54 (46.6\%) had subtotal resection. 93 (80.2\%) patients received postoperative primary radiotherapy (RT), 64 (55.2\%) patients received postoperative primary chemotherapy (CHT).

The comparison of preoperative hematological markers between WNT, SHH, Group 3, and Group 4 MB. We compared the preoperative hematological markers among $144 \mathrm{MB}$ patients by One-way ANOVA. It revealed that NLR $(P<0.01$, Fig. $1 \mathrm{~A}, P<0.05$, Fig. $1 \mathrm{~A})$ in Group $3 \mathrm{MB}$ were significantly higher than those in WNT and SHH MB. The levels of PLR $(P<0.05$, Fig. 1 B, $P<0.05$, Fig. 1B) in Group 3 and Group 4 MB were higher than those in WNT MB (Supplementary Table 1).

Preoperative NLR and PLR were the independent prognostic markers in MB patients. We used the X-tile 3.6.1 software to calculate the cutoff values, and the cutoff values of the preoperative NLR, PLR, MLR, MPV, PDW and AGR for progression free survival (PFS) and overall survival (OS) were listed in Tables 1 and 2. Following $116 \mathrm{MB}$ patients were analysed by univariate analysis (Supplementary Fig. 1). High preoperative $\operatorname{NLR}(P=0.004, P<0.001$, Tables 1 and 2 , Figs $2 \mathrm{~A}$ and $3 \mathrm{~A})$ predicted unfavorable PFS and OS in MB patients. Similarly, high preoperative PLR $(P=0.028, P=0.003$, Tables 1 and 2 , Figs $2 B$ and $3 B$ ) was correlated with unfavorable PFS and OS in patients with MB. Subtotal resection $(P<0.001, P<0.001$, Tables 1 and 2$)$ and no RT $(P=0.003, P<0.001$, Tables 1 and 2$)$ were significantly associated with unfavorable PFS and OS with patients in MB. The tumors located in midline predicted poor PFS $(P=0.048$, Table 1$)$ in patients with MB.

In multivariate analysis, we analyzed preoperative NLR and PLR separately because they were strongly correlated and interfered with each other ${ }^{13}$. The results revealed that preoperative NLR (PFS, $P=0.029$, OS, $P=0.005$, Table 3), preoperative PLR (OS, $P=0.012$, Table 4), the extent of resection (PFS, $P=0.012$, OS, $P=0.011$, Table 3, PFS, $P=0.014$, OS, $P=0.014$, Table 4) and RT (PFS, $P=0.009$, OS, $P=0.001$, Table 3 , PFS, $P=0.010$, OS, $P=0.001$, Table 4) were the independent prognostic factors for the MB patients. When the factor of molecular subgroups was added in multivariate analyses, preoperative NLR lost independent significance in the multivariate analysis for PFS $(P=0.090$, Supplementary Table 2$)$. After adjusting for molecular subgroups, preoperative NLR and PLR were still the independent prognostic factors (OS, $P=0.013$, Supplementary Table 2, OS, $P=0.014$, Supplementary Table 3).

Survival analysis of preoperative NLR and PLR in childhood and adult MB patients. Survival analysis was performed in 93 cases childhood $\mathrm{MB}$ patients and 23 cases adult $\mathrm{MB}$ patients. The results revealed that high preoperative NLR (PFS, $P=0.002$, Fig. $4 \mathrm{~A}$, OS, $P<0.001$, Fig. $4 \mathrm{~B}$ ) and PLR (PFS, $P=0.030$, Fig. $4 \mathrm{C}$, OS, $P=0.003$, Fig. 4D) predicted worse prognosis in childhood MB patients. However, the levels of preoperative NLR and PLR had no prognostic value for PFS $(P=0.686$, Fig. $4 \mathrm{E}, P=0.527$, Fig. $4 \mathrm{G})$ and $\mathrm{OS}(P=0.331$, Fig. 4F, $P=0.588$, Fig. $4 \mathrm{H})$ in adult $\mathrm{MB}$ patients.

Survival analysis of preoperative NLR and PLR in molecular subgroups. There were 23 WNT, 24 $\mathrm{SHH}, 48$ Group 3, and 21 Group $4 \mathrm{MB}$ among $116 \mathrm{MB}$ tumors. When we analysed the survival by restricting the cohort to only these patients, the preoperative NLR in WNT MB were less than 4.94. They were excluded in the survival analysis. It revealed that high preoperative NLR predicted unfavorable OS $(P=0.032$, Fig. $5 \mathrm{G})$ in Group 


\begin{tabular}{|c|c|c|c|}
\hline Parameters & No. of cases & 5-year PFS (\%) & $P$-value \\
\hline \multicolumn{4}{|l|}{ Sex } \\
\hline Male & $75(64.7 \%)$ & 48 & 0.801 \\
\hline Female & $41(35.3 \%)$ & 49.2 & \\
\hline \multicolumn{4}{|l|}{ Age } \\
\hline Children & $93(80.2 \%)$ & 43.3 & 0.109 \\
\hline Adults & $23(19.8 \%)$ & 70.6 & \\
\hline \multicolumn{4}{|l|}{ PreKPS } \\
\hline$<80$ & $37(31.9 \%)$ & 38.2 & 0.387 \\
\hline$\geq 80$ & $79(68.1 \%)$ & 52.1 & \\
\hline \multicolumn{4}{|l|}{ Location } \\
\hline Midline & $88(75.9 \%)$ & 39.1 & 0.048 \\
\hline Lateral & $28(24.1 \%)$ & 70.4 & \\
\hline \multicolumn{4}{|c|}{ Extent of resection } \\
\hline Gross Total & $62(53.4 \%)$ & 71.0 & $<0.001$ \\
\hline Subtotal & $54(46.6 \%)$ & 24.4 & \\
\hline \multicolumn{4}{|l|}{ RT } \\
\hline Yes & $93(80.2 \%)$ & 52.4 & 0.003 \\
\hline No & $23(19.8 \%)$ & 31.5 & \\
\hline \multicolumn{4}{|l|}{$\mathrm{CHT}$} \\
\hline Yes & $64(55.2 \%)$ & 46.9 & 0.564 \\
\hline No & $52(44.8 \%)$ & 51.8 & \\
\hline \multicolumn{4}{|l|}{ NLR } \\
\hline $\mathrm{NLR} \leq 4.94$ & $102(87.9 \%)$ & 51.3 & 0.004 \\
\hline $\mathrm{NLR}>4.94$ & $14(12.1 \%)$ & 28.6 & \\
\hline \multicolumn{4}{|l|}{ PLR } \\
\hline $\mathrm{PLR} \leq 142.31$ & $72(62.1 \%)$ & 55.0 & 0.028 \\
\hline PLR $>142.31$ & $44(37.9 \%)$ & 38.5 & \\
\hline \multicolumn{4}{|l|}{ MLR } \\
\hline MLR $\leq 0.33$ & $102(87.9 \%)$ & 49.8 & 0.157 \\
\hline MLR $>0.33$ & $14(12.1 \%)$ & 39.2 & \\
\hline \multicolumn{4}{|l|}{ MPV } \\
\hline $\mathrm{MPV} \leq 8.80$ & $88(75.9 \%)$ & 44.6 & 0.303 \\
\hline $\mathrm{MPV}>8.80$ & $28(24.1 \%)$ & 60.0 & \\
\hline \multicolumn{4}{|l|}{ PDW } \\
\hline $\mathrm{PDW} \leq 15.90$ & $43(37.1 \%)$ & 37.8 & 0.096 \\
\hline PDW $>15.90$ & $73(62.9 \%)$ & 56.0 & \\
\hline \multicolumn{4}{|l|}{ AGR } \\
\hline $\mathrm{AGR} \leq 1.59$ & $22(19.0 \%)$ & 32.9 & 0.22 \\
\hline AGR $>1.59$ & $94(81.0 \%)$ & 52.7 & \\
\hline
\end{tabular}

Table 1. Univariate analysis of prognostic parameters for PFS in medulloblastoma patients $(\mathrm{n}=116)$. PreKPS: preoperative karnofsky performance status scale. RT: postoperative primary radiotherapy. CHT: postoperative primary chemotherapy. NLR: neutrophil-to-lymphocyte ratio. PLR: platelet-to-lymphocyte ratio. MLR: monocyte-to-lymphocyte ratio. MPV: mean platelet volume. PDW: platelet distribution width. AGR: preoperative albumin-to-globulin ratio.

3 MB. Similarly, the prognosis of Group $4 \mathrm{MB}$ with high preoperative NLR (OS, $P=0.027$, Fig. $5 \mathrm{~K}$ ) and PLR (PFS, $P=0.012$, Fig. 5J, OS, $P=0.009$, Fig. 5L) were worse than those of Group 4 MB with low NLR and PLR.

\section{Discussion}

Recently, numerous studies have revealed that the preoperative hematological markers played important roles in predicting the prognosis of various tumors ${ }^{4}$. For the first time, we investigated the relationship between preoperative hematological markers and the prognosis of $\mathrm{MB}$ patients. We found that high preoperative NLR and PLR predicted unfavorable survival in MB patients, while preoperative MLR, MPV, PDW and AGR had no predictive value on the prognosis in MB patients. In the following multivariate analysis, preoperative NLR and PLR were revealed as independent prognostic factors for the $\mathrm{MB}$ patients. Furthermore, we firstly revealed that the levels of preoperative NLR and PLR in Group 3 MB were higher than those in WNT MB. Subsequent survival analysis demonstrated that high preoperative NLR predicted unfavorable OS in Group 3 and Group 4 MB and high preoperative PLR was associated with unfavorable PFS and OS in Group 4 MB. 


\begin{tabular}{|c|c|c|c|}
\hline Parameters & No. of cases & 5-year OS (\%) & $P$-value \\
\hline \multicolumn{4}{|l|}{ Sex } \\
\hline Male & $75(64.7 \%)$ & 52.2 & 0.242 \\
\hline Female & $41(35.3 \%)$ & 69.9 & \\
\hline \multicolumn{4}{|l|}{ Age } \\
\hline Children & $93(80.2 \%)$ & 51.6 & 0.096 \\
\hline Adults & $23(19.8 \%)$ & 79.1 & \\
\hline \multicolumn{4}{|l|}{ PreKPS } \\
\hline$<80$ & $37(31.9 \%)$ & 54.6 & 0.938 \\
\hline$\geq 80$ & $79(68.1 \%)$ & 59.4 & \\
\hline \multicolumn{4}{|l|}{ Location } \\
\hline Midline & $88(75.9 \%)$ & 49.7 & 0.108 \\
\hline Lateral & $28(24.1 \%)$ & 77.1 & \\
\hline \multicolumn{4}{|c|}{ Extent of resection } \\
\hline Gross Total & $62(53.4 \%)$ & 81.6 & $<\mathbf{0 . 0 0 1}$ \\
\hline Subtotal & $54(46.6 \%)$ & 32.8 & \\
\hline \multicolumn{4}{|l|}{ RT } \\
\hline Yes & $93(80.2 \%)$ & 64.1 & $<\mathbf{0 . 0 0 1}$ \\
\hline No & $23(19.8 \%)$ & 32.8 & \\
\hline \multicolumn{4}{|l|}{$\mathrm{CHT}$} \\
\hline Yes & $64(55.2 \%)$ & 56 & 0.469 \\
\hline No & $52(44.8 \%)$ & 63.7 & \\
\hline \multicolumn{4}{|l|}{ NLR } \\
\hline $\mathrm{NLR} \leq 4.94$ & $102(87.9 \%)$ & 62.5 & $<\mathbf{0 . 0 0 1}$ \\
\hline $\mathrm{NLR}>4.94$ & $14(12.1 \%)$ & 27.8 & \\
\hline \multicolumn{4}{|l|}{ PLR } \\
\hline $\mathrm{PLR} \leq 147.50$ & $77(66.4 \%)$ & 69.9 & 0.003 \\
\hline $\mathrm{PLR}>147.50$ & $39(33.6 \%)$ & 38.8 & \\
\hline \multicolumn{4}{|l|}{ MLR } \\
\hline$M L R \leq 0.12$ & $12(10.3 \%)$ & 47.6 & 0.124 \\
\hline MLR > 0.12 & $104(89.7 \%)$ & 58.2 & \\
\hline \multicolumn{4}{|l|}{ MPV } \\
\hline $\mathrm{MPV} \leq 8.00$ & $51(44.0 \%)$ & 46 & 0.625 \\
\hline $\mathrm{MPV}>8.00$ & $65(56.0 \%)$ & 64.9 & \\
\hline \multicolumn{4}{|l|}{ PDW } \\
\hline $\mathrm{PDW} \leq 15.90$ & $43(37.1 \%)$ & 43.2 & 0.052 \\
\hline $\mathrm{PDW}>15.90$ & $73(62.9 \%)$ & 69.1 & \\
\hline \multicolumn{4}{|l|}{ AGR } \\
\hline AGR $\leq 1.59$ & $22(19.0 \%)$ & 38 & 0.053 \\
\hline AGR > 1.59 & $94(81.0 \%)$ & 63.5 & \\
\hline
\end{tabular}

Table 2. Univariate analysis of prognostic parameters for OS in medulloblastoma patients $(\mathrm{n}=116)$. PreKPS: preoperative karnofsky performance status scale. RT: postoperative primary radiotherapy. CHT: postoperative primary chemotherapy. NLR: neutrophil-to-lymphocyte ratio. PLR: platelet-to-lymphocyte ratio. MLR: monocyte-to-lymphocyte ratio. MPV: mean platelet volume. PDW: platelet distribution width. AGR: preoperative albumin-to-globulin ratio.

The exact mechanism about the role of preoperative NLR in the prognosis of tumors need to be fully elucidated. Elevated NLR means more neutrophils, fewer lymphocytes, or both. On the one hand, elevated neutrophils could promote tumor invasion and metastasis by releasing many reactive oxygen species ${ }^{14}$ and cytokines including interleukin-1 (IL-1), tumor necrosis factor (TNF) and vascular endothelial growth factor (VEGF), etc. $^{15,16}$. The reactive oxygen species could induce the DNA damage and genetic instability, and the cytokines promoted to tumor angiogenesis, proliferation and metastasis ${ }^{17,18}$. On the other hand, the tumor infiltrating lymphocytes (TILs) which were differentiated from the T-lymphocytes were considered as systemic and local indicators of anti-tumor reaction. Reduced of TILs were revealed associated with poor prognosis in tumors ${ }^{19}$. A recent study revealed that a decreased infiltrating CD8 + T lymphocyte predicted poor prognosis in patients with $\mathrm{MB}^{20}$. Therefore, elevated NLR may indicate the poor prognosis of the patients with MB. In addition, we also found that preoperative NLR in Group 3 MB were significantly higher than those in WNT MB. This finding was in accordance with that the prognosis of WNT MB were significantly better than those in Group $3 \mathrm{MB}$. In addition, high 

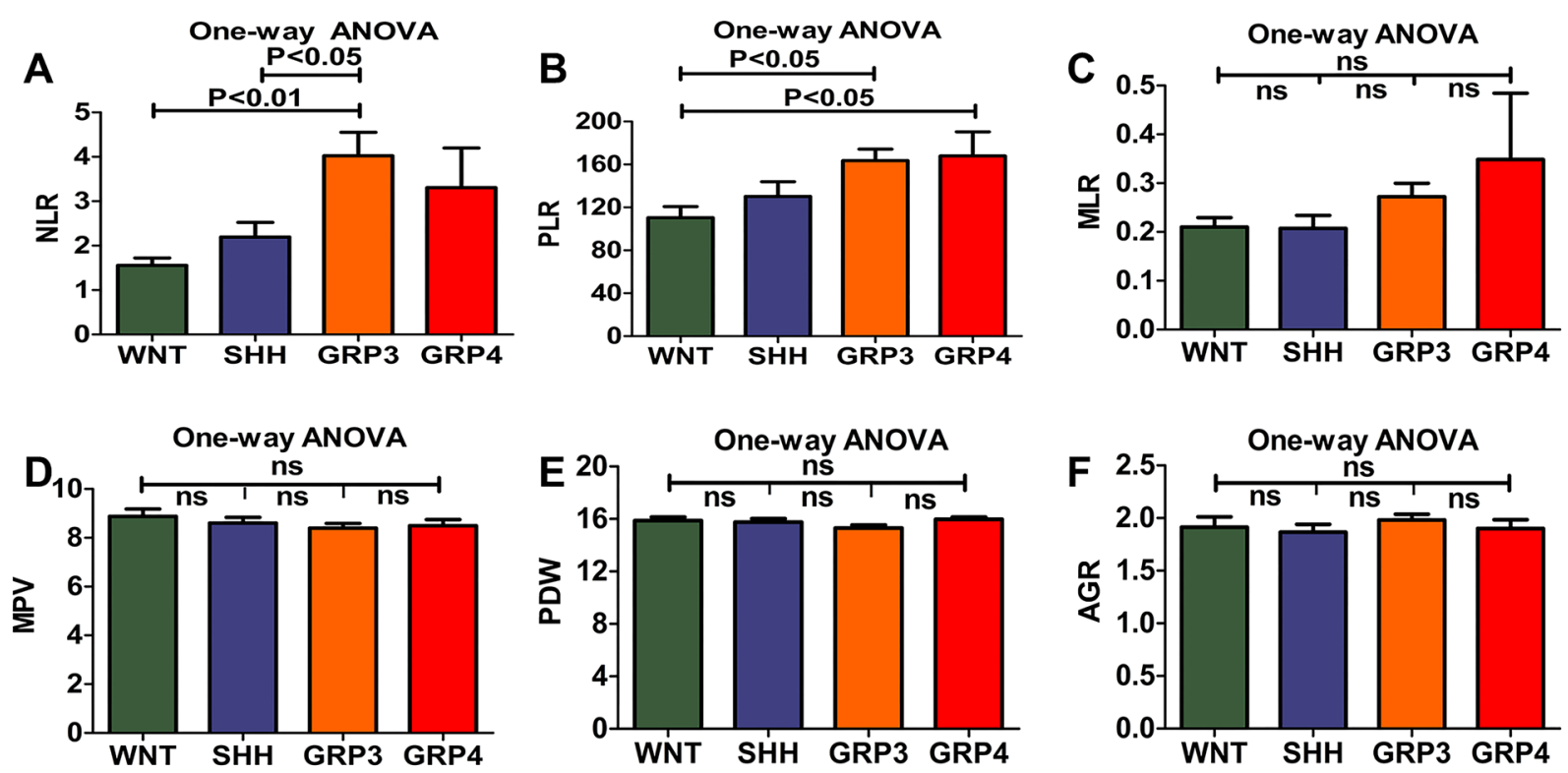

Figure 1. The levels of preoperative NLR, PLR, MLR, MPV, PDW and AGR presented among WNT, SHH, Group 3, and Group 4 were analyzed by One-way ANOVA. (A) The levels of preoperative NLR in Group $3 \mathrm{MB}$ were significantly higher than those in WNT $(\mathrm{P}<0.01)$ and SHH MB $(\mathrm{P}<0.05)$. (B) The levels of preoperative PLR in Group $3(\mathrm{P}<0.05)$ and Group $4 \mathrm{MB}(\mathrm{P}<0.05)$ were higher than those in WNT MB. $(\mathbf{C}-\mathbf{F})$ The levels of preoperative MLR, MPV, PDW, and AGR among WNT, SHH, Group 3, and Group 4 had no statistically significant.
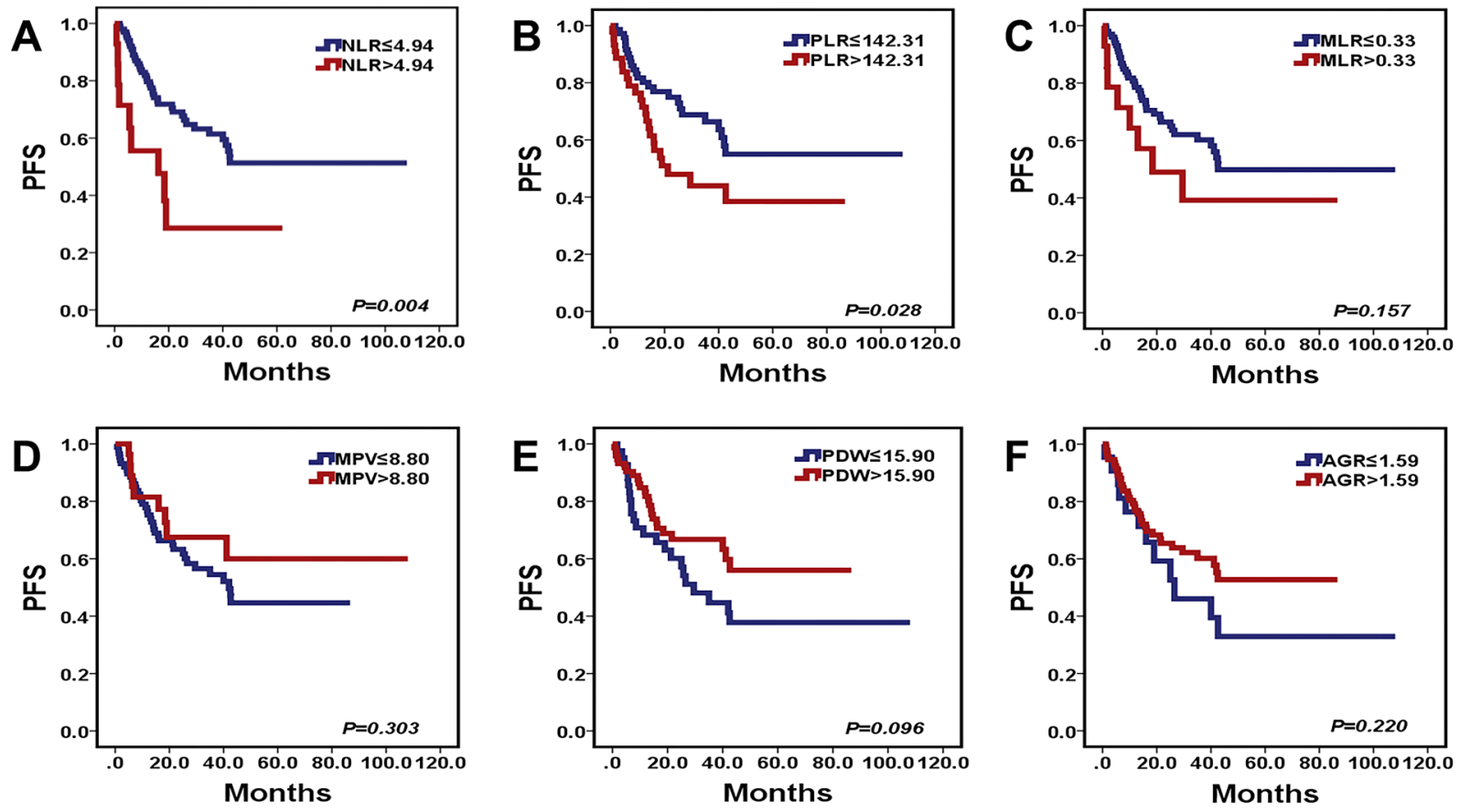

Figure 2. Kaplan-Meier survival curves for PFS probability according to preoperative NLR, PLR, MLR, MPV, PDW, and AGR levels. $(\mathbf{A}, \mathbf{B})$ High preoperative NLR $(P=0.004)$ and PLR $(P=0.028)$ predicted short PFS in patients with MB. (C-F) The levels of preoperative MLR, MPV, PDW, and AGR exited no statistically significant for PFS $(P=0.157, P=0.303, P=0.096, P=0.220)$ in patients with $\mathrm{MB}$.

preoperative NLR was revealed associated with short OS in Group 3 and Group 4 MB. These findings indicated the differential prognostic significances of preoperative NLR in the four molecular subgroups of MB.

The preoperative PLR was also revealed to play an important role in predicting the prognosis in patients with MB. It had been revealed the platelet receptors such as GP1b/IX/V, P-selectin and alphaIIb-beta3 integrin 

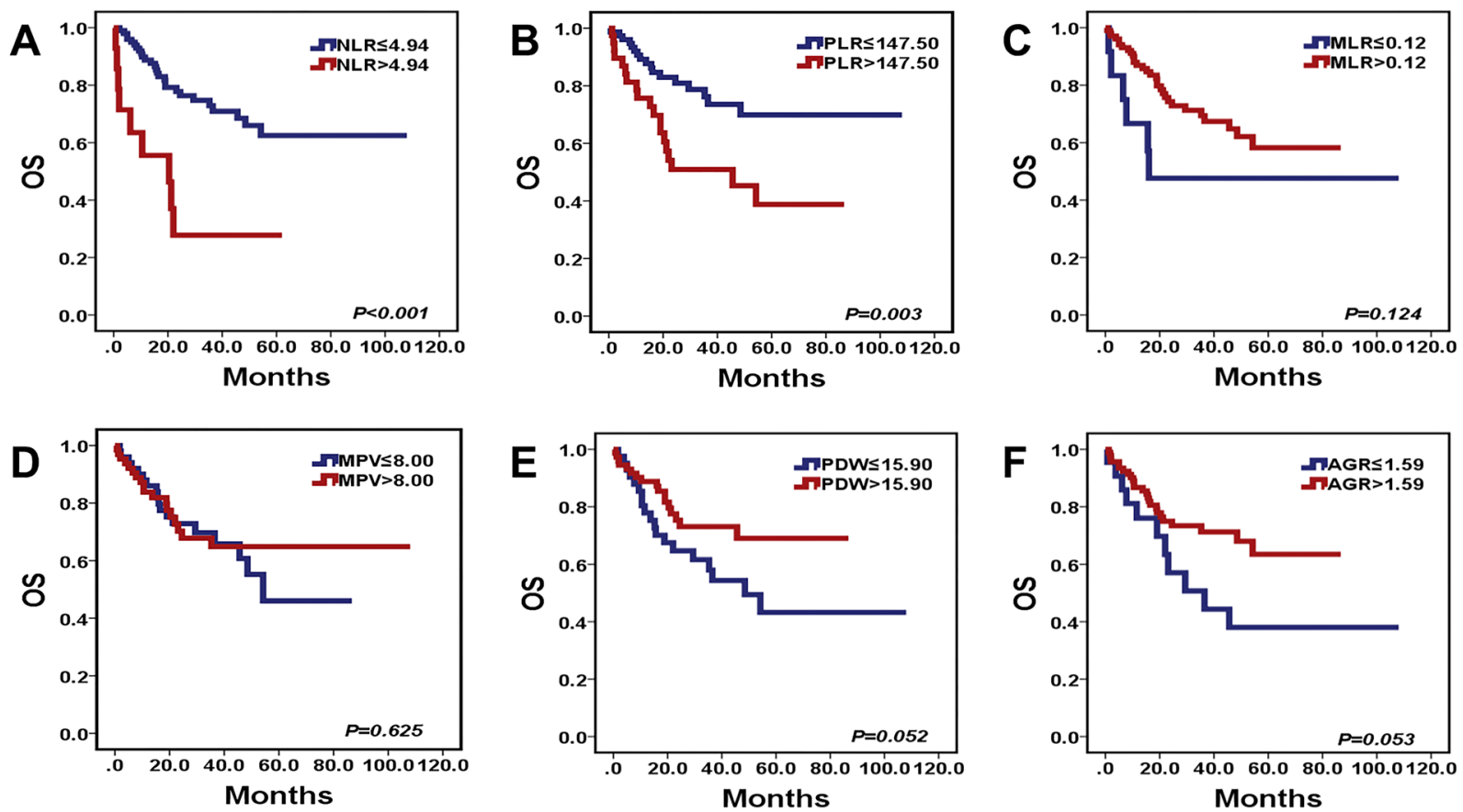

Figure 3. Kaplan-Meier survival curves for OS probability according to preoperative NLR, PLR, MLR, MPV, PDW and AGR levels. (A,B) High preoperative NLR and PLR predicted short OS $(P<0.001, P=0.003)$ in patients with MB. (C-F) The levels of preoperative MLR, MPV, PDW, and AGR had no prognostic value for OS $(P=0.124, P=0.625, P=0.052, P=0.053)$ in patients with MB.

\begin{tabular}{|c|c|c|c|c|}
\hline \multirow[b]{2}{*}{ Parameters } & \multicolumn{2}{|l|}{ PFS } & \multicolumn{2}{|l|}{ OS } \\
\hline & OR $(95 \% C I)$ & $P$-value & OR $(95 \% \mathrm{CI})$ & $P$-value \\
\hline \multicolumn{5}{|l|}{ Age } \\
\hline Children & Reference & & Reference & \\
\hline Adults & $0.965(0.372-2.504)$ & 0.942 & $0.994(0.306-3.233)$ & 0.992 \\
\hline \multicolumn{5}{|c|}{ Extent of resection } \\
\hline Gross Total & Reference & & Reference & \\
\hline Subtotal & $2.337(1.201-4.546)$ & 0.012 & $2.895(1.269-6.601)$ & 0.011 \\
\hline \multicolumn{5}{|l|}{ RT } \\
\hline Yes & Reference & & Reference & \\
\hline No & $2.415(1.241-4.702)$ & 0.009 & $3.420(1.635-7.153)$ & 0.001 \\
\hline \multicolumn{5}{|l|}{ Location } \\
\hline Midline & Reference & & Reference & \\
\hline Lateral & $0.668(0.294-1.513)$ & 0.333 & $0.760(0.296-1.955)$ & 0.570 \\
\hline NLR & $1.046(1.005-1.089)$ & 0.029 & $1.061(1.018-1.106)$ & 0.005 \\
\hline
\end{tabular}

Table 3. Multivariate analysis of prognostic parameters for PFS and OS in medulloblastoma patients $(n=116)$. OR: odds ratio. CI: confidence interval. RT: postoperative primary radiotherapy. NLR: neutrophil-to-

lymphocyte ratio.

were associated with various tumor progression and metastasis. Additionally, the platelets could release over 30 important angiogenesis regulating proteins in which VEGF was the most important ${ }^{21}$. The levels of VEGF mRNA in Group 3 subgroup (which prognosis is the worst) were significantly higher than those with the other subgroups in patients with $\mathrm{MB}^{22}$. Furthermore, the platelets activation and platelets related-protein contributed to the inflammatory response, which could lead to neutrophilia, leukocytosis, thrombocytosis and lymphocytope$\mathrm{nia}^{23,24}$. And the platelets protein was reported to enhance the tumor growth and metastasis ${ }^{21}$. Xia, et al. and Yun, et al. reported that the pretreatment PLR was an independent risk factor for OS in patients with osteosarcoma and renal cell carcinoma ${ }^{4,24}$. In our study, high preoperative PLR was associated with poor PFS and OS in MB patients, particularly high preoperative PLR predicted poor PFS and OS in Group 4 MB.

There exist controversies in the prognostic roles of preoperative MLR, MPV, PDW and AGR in cancers. For example, high preoperative MLR was associated with poor survival in colorectal cancer ${ }^{3}$ and esophageal can$\operatorname{cer}^{2}$. However, a recent study revealed that preoperative MLR exhibited no prognostic value in glioblastoma ${ }^{13}$. Similarly, recent studies demonstrated that decreased preoperative MPV had a relationship with unfavorable 


\begin{tabular}{|c|c|c|c|c|}
\hline \multirow[b]{2}{*}{ Parameters } & \multicolumn{2}{|l|}{ PFS } & \multicolumn{2}{|l|}{ OS } \\
\hline & OR $(95 \% C I)$ & $P$-value & OR $(95 \% C I)$ & $P$-value \\
\hline \multicolumn{5}{|l|}{ Age } \\
\hline Children & Reference & & Reference & \\
\hline Adults & $0.990(0.389-2.615)$ & 0.986 & $0.965(0.327-3.462)$ & 0.917 \\
\hline \multicolumn{5}{|c|}{ Extent of resection } \\
\hline Gross Total & Reference & & Reference & \\
\hline Subtotal & $2.313(1.188-4.504)$ & 0.014 & $2.817(1.233-6.438)$ & 0.014 \\
\hline \multicolumn{5}{|l|}{ RT } \\
\hline Yes & Reference & & Reference & \\
\hline No & $2.419(1.240-4.719)$ & 0.010 & $3.478(1.657-7.300)$ & 0.001 \\
\hline \multicolumn{5}{|l|}{ Location } \\
\hline Midline & Reference & & Reference & \\
\hline Lateral & $0.625(0.276-1.413)$ & 0.259 & $0.689(0.269-1.762)$ & 0.437 \\
\hline PLR & $1.002(0.997-1.004)$ & 0.069 & $1.002(1.001-1.004)$ & 0.012 \\
\hline
\end{tabular}

Table 4. Multivariate analysis of prognostic parameters for PFS and OS in medulloblastoma patients $(n=116)$. OR: odds ratio. CI: confidence interval. RT: postoperative primary radiotherapy. PLR: platelet-to-lymphocyte ratio.

prognosis of the renal cell carcinoma ${ }^{9}$ and gliomas ${ }^{25}$. On the contrary, a study revealed that high preoperative MPV had poor prognosis in patients with lung cancer ${ }^{26}$. Additionally, Zhang, et al. illustrated that decreased preoperative PDW had an unfavorable prognosis in stomach cancer ${ }^{10}$. However, other researches revealed that high preoperative PDW had unfavorable prognosis in laryngeal cancer ${ }^{27}$ and melanoma ${ }^{28}$. Previous studies showed that low preoperative AGR had unfavorable OS in breast cancer and colorectal cancer ${ }^{29,30}$. Our study revealed that preoperative MLR, MPV, PDW and AGR had no prognostic value in MB patients, and the levels of preoperative MLR, MPV, PDW, and AGR among WNT, SHH, Group 3, and Group 4 MB had no significant differences.

Previous study ${ }^{31}$ reported that gross total tumor resection predicted better PFS and OS in MB patients. In our study, the gross total tumor resection correlated with more favorable PFS and OS in our series in the univariate analysis. Moreover, the extent of resection was the independent prognostic factor in $\mathrm{MB}$ patients in the multivariate analysis. Therefore, our results corroborated that the extent of resection is one of the most significant predictors of PFS and OS in MB patients.

In the multivariate analyses without molecular subgroups, preoperative NLR and PLR were the independent prognostic factors for PFS and OS in MB patients. When molecular subgroups were added in multivariate analyses, preoperative NLR lost independent significance in the multivariate analysis for PFS. After adding molecular subgroup in the multivariate analyses, preoperative NLR and PLR were still the independent prognostic factors for OS. The OS could be influenced by the differences in the protocols of salvage treatment after tumor recurrence and this might confuse the prognostic significance of hematological markers in MB. Our results indicate preoperative NLR may be influenced by molecular subgroups in PFS. Future cohorts with relatively homogeneous salvage adjuvant therapies and larger sample size are needed to further clarify this question.

In our opinion, we think a One-way ANOVA model with pairwise comparison is appropriate for comparing across multiple groups here. Whereas a One-way ANOVA model assesses whether a significant difference exists among all the groups, pairwise comparisons can be used to determine which groups' differences are statistically significant. Eckel-Passow, J. E. et al. ${ }^{32}$ previously used pairwise comparisons in One-way ANOVA statistics in their paper.

Admittedly, some limitations existed in present study. Firstly, the incidence of MB is low. Some studies reported that the estimated incidence of $\mathrm{MB}$ in children was about $0.5 / 100,000^{33,34}$. Moreover, MB represented a rare tumor in adults and comprised less than $1 \%$ of adult primary brain neoplasms ${ }^{35}$. Compared to previous study ${ }^{36}$, the current cohort of $116 \mathrm{MB}$ patients with complete survival data and molecular subgroup information may not be sufficient to draw final conclusions, especially when the cohort was divided into four molecular subgroups. Therefore, these findings revealed by the current study should be interpreted with caution. Secondly, our research was based on a single-center retrospective study and the findings should be corroborated by multi-centre prospective studies. Thirdly, we revealed preoperative NLR and PLR were correlated with the survival of MB patients based on molecular subgroups, but the exact mechanism between the levels of preoperative NLR, PLR and molecular subgroups need further investigation. Last not the least, according to previous studies ${ }^{37,38}$, patients with disseminated MB are classified according to Chang's operative staging system. Due to the lack of Chang classification data, we could not investigate whether high preoperative NLR/PLR predict patients with higher probability of experiencing metastasis of $\mathrm{MB}$ in the current study.

In conclusion, we firstly demonstrated high preoperative NLR and PLR were significantly correlated with poor survivals of patients with MB. Moreover, the levels of preoperative NLR and PLR in Group 3 MB were significantly higher than those in WNT MB. High preoperative NLR and PLR predict unfavorable survival in Group 3 and Group $4 \mathrm{MB}$. These findings indicate preoperative NLR and PLR can be used as prognostic predictors for Group 3 and Group $4 \mathrm{MB}$ patients. 

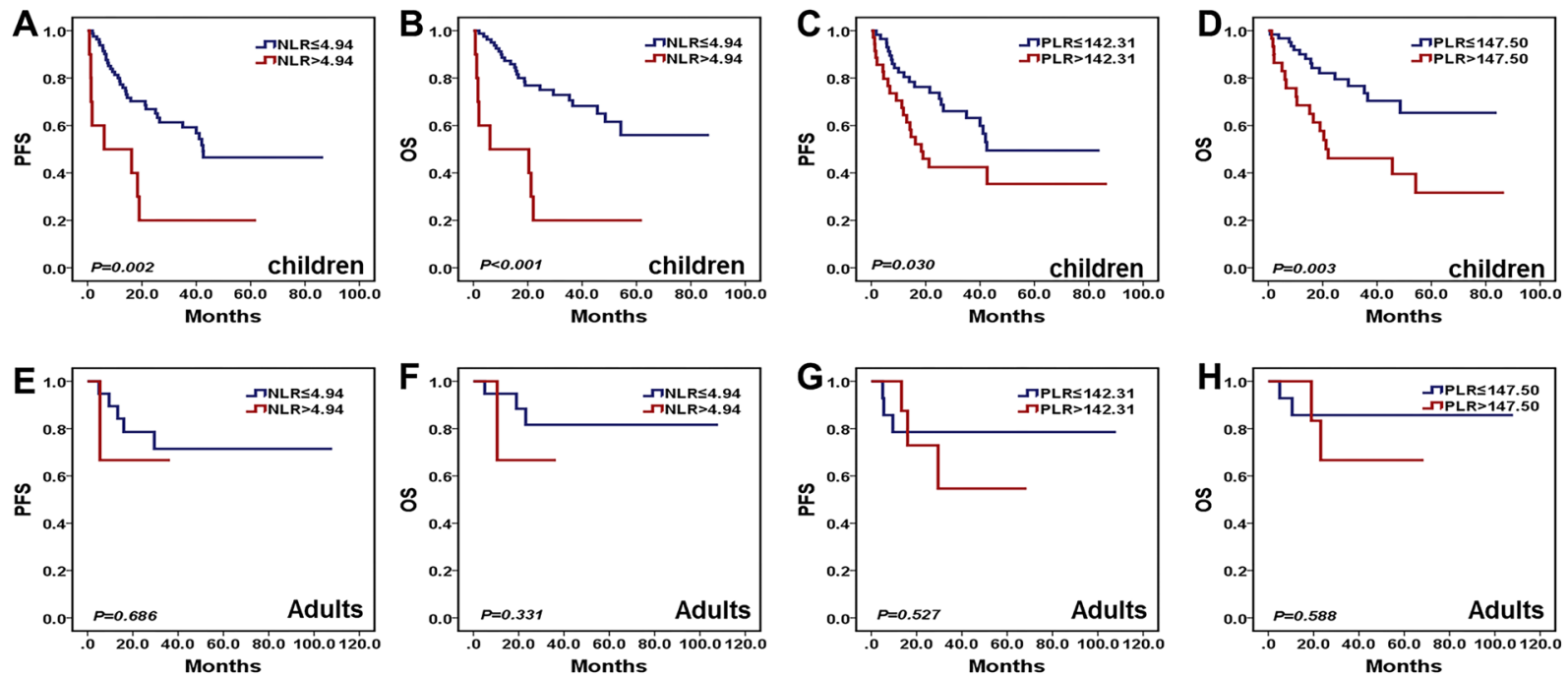

Figure 4. Kaplan-Meier survival curves for PFS and OS probability according to preoperative NLR and PLR in childhood and adult MB patients. (A-D) High preoperative NLR (PFS, $P=0.002$, OS, $P<0.001$ ) and PLR (PFS, $P=0.030$, OS, $P=0.003)$ predicted worse prognosis in childhood MB patients. $(\mathbf{E}-\mathbf{H})$ the levels of preoperative NLR and PLR had no prognostic value for PFS $(P=0.686, P=0.527)$ and $\mathrm{OS}(P=0.331, P=0.588)$ in adult MB patients.
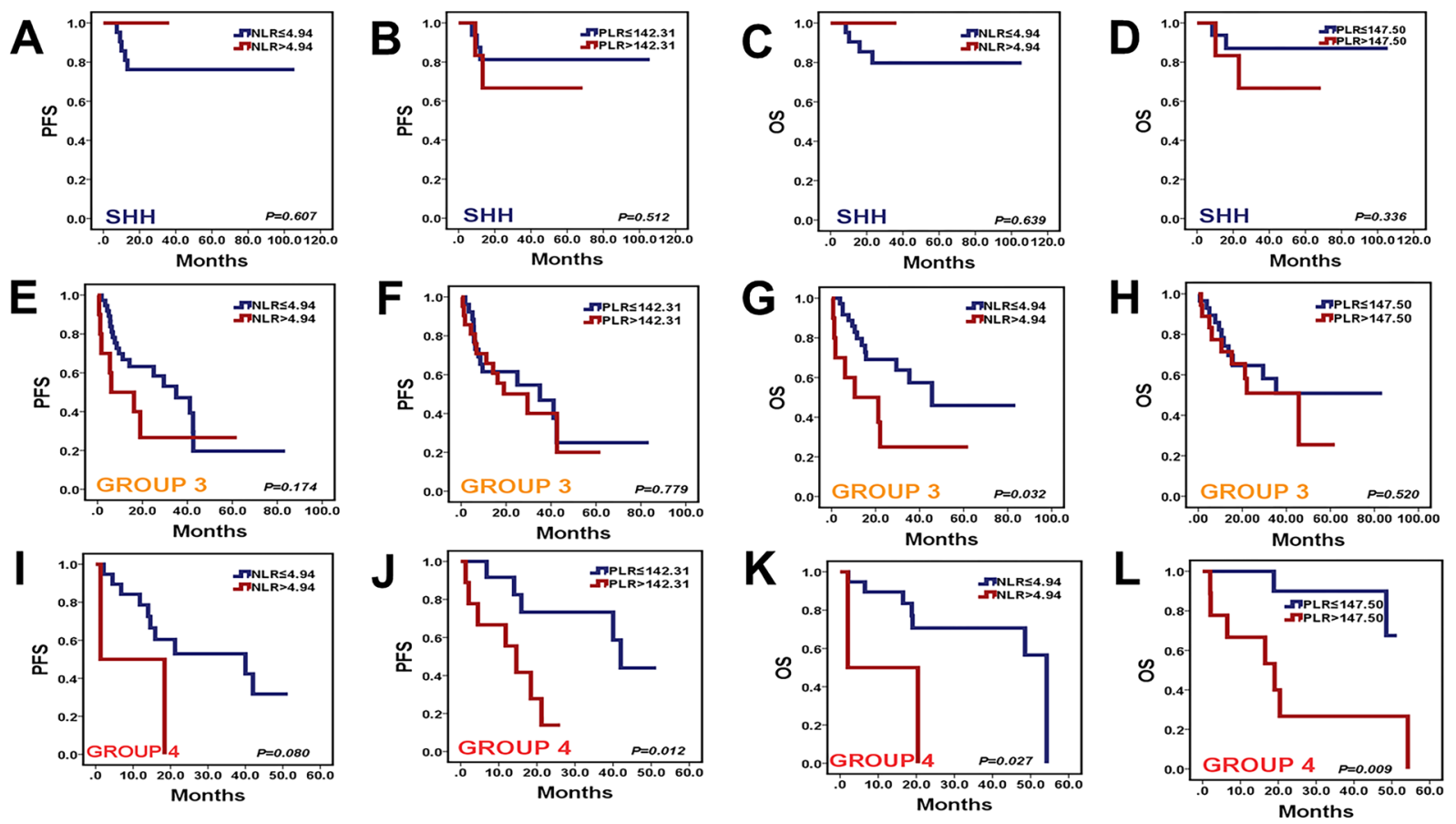

Figure 5. Kaplan-Meier survival curves for PFS and OS probability according to preoperative NLR and PLR levels in SHH, Group 3, and Group 4 MB. (A-D) The levels of preoperative NLR and PLR exited no statistically significant for PFS $(P=0.607, P=0.512)$ and OS $(P=0.639, P=0.336)$ in patients with SHH MB. $(\mathbf{E}, \mathbf{F})$ The levels of preoperative NLR and PLR had no prognostic value for PFS $(P=0.174, P=0.779)$ in Group $3 \mathrm{MB}$. $(G)$ High preoperative NLR predicted poor OS $(P=0.032)$ in Group $3 \mathrm{MB}$. $(\mathbf{H})$ The level of preoperative PLR had no association with OS $(P=0.520)$ in Group $3 \mathrm{MB}$. (I) The level of preoperative NLR exited no statistically significant for PFS $(P=0.080)$ in Group $4 \mathrm{MB}$. (J) High preoperative PLR were associated with poor PFS $(P=0.012)$ in Group 4 MB. $(\mathbf{K})$ High preoperative NLR was associated with poor OS $(P=0.027)$ in Group 4 MB. (L) High preoperative PLR were associated with poor OS $(P=0.009)$ in Group $4 \mathrm{MB}$. 


\section{Materials and Methods}

Study population. 183 patients were surgically treated in the First Affiliated Hospital of Zhengzhou University from January 2009 to January 2018. The diagnosis of MB was confirmed by postoperative pathology. Patients with hematological diseases, serious infections, severe renal, hepatic dysfunction, diabetes mellitus, metabolic syndrome, surgery, trauma, cardiac-cerebral vascular disease, any therapy with anticoagulant, previous history of infection within 3 months and any inflammatory conditions that could significantly influence preoperative hematological markers were excluded. 39 patients were excluded and 144 patients were enrolled in the cohort. In the $144 \mathrm{MB}$ patients, $116 \mathrm{MB}$ patients were successfully followed up, 28 cases were lost to follow up, (Supplementary Fig. 1). Clinical data including gender, age, preoperative KPS value, tumor location, extent of resection, RT, and CHT were collected from medical records. The follow-up data of the cohort were acquired by telephone follow up or outpatient clinic records. OS was defined as the interval between surgery and death or the end of follow up. PFS was measured from the date of diagnosis to the date of disease recurrence, death, or last follow up.

Preoperative hematological markers. $144 \mathrm{MB}$ patients' routine blood test and liver function were obtained preoperatively before any treatment. The blood count included blood neutrophil, lymphocyte, platelet, monocyte, MPV, and PDW. The liver function included albumin count, and globulin count. The NLR was equal to the absolute neutrophil count divided by the absolute lymphocyte count, the PLR was equal to the absolute platelet count divided by the absolute lymphocyte count, the MLR was defined as the absolute monocyte count divided by the absolute lymphocyte count, and the AGR was defined as the albumin count divided by globulin count.

Determination of Molecular subgroups. 183 Formalin-fixed paraffin embedded (FFPE) tissues from tumor resection were available in all the cases. RNA was extracted from FFPE tissues, then a nanoString-based assay was employed to test the tumor samples for detection of molecular subgroups according to the Northcott, P. A. et al. ${ }^{39}$ previously described.

Statistical methods. Statistical analyses were performed using SPSS 21.0 (IBM Crop, Armonk, NY, USA), Graph-Pad Prism 5.0 (Graph-Pad Inc, La Jolla, USA) and X-tile 3.6.1 (http://medicine.yale.edu/lab/rimm/ research/software.aspx). The differences of preoperative hematological markers between WNT, SHH, Group 3, and Group 4 were compared by One-way ANOVA. Kaplan-Meier method was used to calculate survival curves. The comparison of survival rates in different groups were conducted by the Log-rank test (univariate analysis). Cox proportional hazards regression model was used to evaluate independent prognostic factors (multivariate analysis). Values of $P<0.05$ were considered as statistically significant.

Our research was approved by the Human Scientific Ethics Committee of the First Affiliated Hospital of Zhengzhou University. All procedures performed in studies involving human participants were in accordance with the ethical standards of the institutional and national research committee and with the 1975 Helsinki declaration and its later amendments or comparable ethical standards. An informed consent was obtained from all individual participants included in the study.

\section{Data Availability}

The datasets generated during and/or analyzed during the current study are available from the corresponding author on reasonable request.

\section{References}

1. Zhang, Z. Y. et al. Clinicopathological analysis of UHRF1 expression in medulloblastoma tissues and its regulation on tumor cell proliferation. Med Oncol. 33(9), 99 (2016).

2. Hirahara, N., Matsubara, T., Mizota, Y., Ishibashi, S. \& Tajima, Y. Prognostic value of preoperative inflammatory response biomarkers in patients with esophageal cancer who undergo a curative thoracoscopic esophageltomy. BMC Surgery. 16(1), 66-78 (2016).

3. Wu, Q. et al. Prognostic role of the lymphocyte-to-monocyte ratio in colorectal cancer: An up-to-date meta-analysis. Medicine (Baltimore). 96(22), e7051 (2017).

4. Hu, H. et al. Prognostic value of preoperative NLR, dNLR, PLR, CRP in surgical renal cell carcinoma patients. World J Urol. 35(2), 261-270 (2017)

5. Han, S. et al. Pre-treatment neutrophil-to-lymphocyte ratio is associated with neutrophil and T-cell infiltration and predicts clinical outcome in patients with glioblastoma. BMC Cancer 15, 617-717 (2015).

6. Cho, I. R. et al. Pre-treatment neutrophil to lymphocyte ratio as a prognostic marker to predict chemotherapeutic response and survival outcomes in metastatic advanced gastric cancer. Gastric Cancer. 17(4), 703-10 (2014).

7. Al Murri, A. M. et al. Evaluation of the relationship between the systemic inflammatory response and cancer-specific survival in patients with primary operable breast cancer. Br J Cancer. 96(6), 891-5 (2007).

8. Teng, J. J. et al. Prognostic value of peripheral blood lymphocyte-to-monocyte ratio in patients with solid tumors: a meta-analysis. Onco Targets Ther. 9, 37-47 (2015).

9. Yun, Z. Y. et al. Association of decreased mean platelet volume with renal cell carcinoma. Int J Clin Oncol. 22(6), 1076-1080 (2017).

10. Zhang, X. et al. Platelet distribution width correlates with prognosis of gastric cancer. Oncotarget. 8(12), 20213-20219 (2017).

11. Robinson, G. et al. Novel mutations target distinct subgroups of medulloblastoma. Nature. 488(7409), 43-8 (2012).

12. Louis, D. N. et al. The 2016 world health organization classification of tumors of the central nervous system: a summary. Acta Neuropathol. 131, 803-820 (2016).

13. Wang, P. F. et al. Preoperative inflammation markers and IDH mutation status predict glioblastoma patient survival. Oncotarget. 8(30), 50117-50123 (2017).

14. Muller, I. et al. Polymorphonuclear neutrophils and T lymphocytes: strange bedfellows or brothers in arms? Trends Immunol. 30(11), 522-30 (2009).

15. Kuper, H. et al. Infections as a major preventable cause of human cancer. J Intern Med. 248(3), 171-83 (2000).

16. Cho, H. et al. Pre-treatment neutrophil to lymphocyte ratio is elevated in epithelial ovarian cancer and predicts survival after treatment. Cancer Immunol Immunother. 58(1), 15-23 (2009). 
17. Kusumanto, Y. H. et al. Platelets and granulocytes, in particular the neutrophils, form important compartments for circulating vascular endothelial growth factor. Angiogenesis. 6(4), 283-7 (2003).

18. Shacter, E. I. et al. Chronic Inflammation and Cancer. Oncology. 16(2), 217-2326299 (2002).

19. Vermeulen, J. F. et al. Prognostic relevance of tumor-infiltrating lymphocytes and immune checkpoints in pediatric medulloblastoma. Oncoimmunology. 7(3), e1398877 (2017).

20. Murata, D. et al. High programmed cell death 1 ligand-1 expression: associated with CD8+ T-cell infiltration and poor prognosis in human medulloblastoma. J Neurosurg. 128(3), 710-716 (2018).

21. Bambace, N. M. et al. The platelet contribution to cancer progression. J Thromb Haemost. 9(2), 237-49 (2011).

22. Thompson, E. M. et al. The role of angiogenesis in Group 3 medulloblastoma pathogenesis and survival. Neuro Oncol. 19(9), 1217-1227 (2017).

23. Ikeda, M. et al. Poor prognosis associated with thrombocytosis in patients with gastric cancer. Ann Surg Oncol. 9(3), 287-91 (2002).

24. Xia, W. K. et al. Prognostic performance of pre-treatment NLR and PLR in patients suffering from osteosarcoma. World J Surg Oncol. 14, 127 (2016).

25. Dagistan, Y. et al. Evaluation of simple blood counts as inflammation markers for brain tumor patients. Neurol Neurochir Pol. 50(4), $231-5(2016)$.

26. Omar, M. et al. Role of increased mean platelet volume (MPV) and decreased MPV/platelet count ratio as poor prognostic factors in lung cancer. Clin Respir J. 12(3), 922-929 (2018).

27. Cui, M. M. et al. Platelet distribution width correlates with prognosis of non-small cell lung cancer. Sci Rep. 7(1), 3456 (2017).

28. Li, N. et al. Increased platelet distribution width predicts poor prognosis in melanoma patients. Sci Rep. 7(1), 2970 (2017).

29. Azab, B. N. et al. Value of the pretreatment albumin to globulin ratio in predicting long-term mortality in breast cancer patients. Am J Surg. 206(5), 764-70 (2013).

30. Azab, B. et al. The value of the pretreatment albumin/globulin ratio in predicting the long-term survival in colorectal cancer. Int $J$ Colorectal Dis. 28(12), 1629-36 (2013).

31. Zhang, Z. Y. et al. Medulloblastoma in China: Clinicopathologic Analyses of SHH, WNT, and Non-SHH/WNT Molecular Subgroups Reveal Different Therapeutic Responses to Adjuvant Chemotherapy. PLoS One. 9(6), e99490 (2014).

32. Eckel-Passow, J. E. et al. Glioma Groups Based on 1p/19q, IDH, and TERT Promoter Mutations in Tumors. N Engl J Med. 372(26), 2499-508 (2015)

33. Packer, R. J., Cogen, P., Vezina, G. \& Rorke, L. B. Medulloblastoma: clinical and biologic aspects. Neuro Oncol. 1, 232-250 (1999).

34. Sarkar, C. et al. Are childhood and adult medulloblastomas different? A comparative study of clinicopathological features, proliferation index and apoptotic index. J Neurooncol. 59, 49-61 (2002).

35. Wrensch, M., Minn, Y., Chew, T., Bondy, M. \& Berger, M. S. Epidemiology of primary brain tumors: current concepts and review of the literature. Neuro Oncol. 4, 278-299 (2002).

36. Schwalbe, E. C. et al. Novel molecular subgroups for clinical classification and outcome prediction in childhood medulloblastoma: a cohort study. Lancet Oncol. 18(7), 958-971 (2017).

37. Dufour, C. et al. Metastatic Medulloblastoma in Childhood: Chang's Classification Revisited. Int J Surg Oncol. 2012, 245385 (2012).

38. Chang, C. H., Housepian, E. M. \& Herbert, C. Jr. An operative staging system and a megavoltage radiotherapeutic technic for cerebellar medulloblastomas. Radiology. 93(6), 1351-1359 (1969).

39. Northcott, P. A. et al. Rapid, reliable, and reproducible molecular sub-grouping of clinical medulloblastoma samples. Acta Neuropathol. 123(4), 615-26 (2012).

\section{Acknowledgements}

This work was supported by the following grants: the National Natural Science Foundation of China to Zhen-yu Zhang (81702465), the Science and Technology Program of Henan Province to Zhen-yu Zhang, the Youth Innovation Fund of The First Affiliated Hospital of Zhengzhou University to Zhen-yu Zhang, the Key Research Projects of Henan Higher Education of Wei-wei Wang (No.18A310033), the Science and Technology Program of Henan Province to Jin-qiao Zhou, and the Medical Science and Technology Program of Henan Province to Jinqiao Zhou (201602058). We would like to thank Jie-sheng Lu (Laboratory of Personalized Genomic Medicine, Columbia University in the City of New York, USA) for assistance with reviewing this article.

\section{Author Contributions}

Conceived and designed the experiments: K.L., Z.Y.Z. and Z.F.Z. Performed the experiments: K.L., W.C.D. and H.B.Z. Analyzed the data: K.L. Contributed to writing and reviewing of the manuscript: all authors.

\section{Additional Information \\ Supplementary information accompanies this paper at https://doi.org/10.1038/s41598-019-49733-6.}

Competing Interests: The authors declare no competing interests.

Publisher's note: Springer Nature remains neutral with regard to jurisdictional claims in published maps and institutional affiliations.

Open Access This article is licensed under a Creative Commons Attribution 4.0 International License, which permits use, sharing, adaptation, distribution and reproduction in any medium or format, as long as you give appropriate credit to the original author(s) and the source, provide a link to the Creative Commons license, and indicate if changes were made. The images or other third party material in this article are included in the article's Creative Commons license, unless indicated otherwise in a credit line to the material. If material is not included in the article's Creative Commons license and your intended use is not permitted by statutory regulation or exceeds the permitted use, you will need to obtain permission directly from the copyright holder. To view a copy of this license, visit http://creativecommons.org/licenses/by/4.0/.

(c) The Author(s) 2019 